\title{
LINEAR INDEPENDENCE OF VALUES OF A CERTAIN GENERALIZATION OF THE EXPONENTIAL FUNCTION II
}

ROLF WALLisser

Abstract: The arithmetical nature of values at rational points of the hypergeometric series

$$
{ }_{Q} G_{R}(x):=\sum_{n=0}^{\infty} \frac{R(1) R(2) \cdots R(n)}{Q(1) Q(2) \cdots Q(n)} x^{n}
$$

is studied. $R$ and $Q$ are polynomials with integer coefficients. Using deep results on higher congruences going essentially back to Frobenius, Dedekind, Nagell and Schinzel a measure of $\mathbb{Q}$-linear independence of such values is given.

In contrast to former investigations no preseribed factorisations of the polynomials $Q$ and $R$ are necessary. Here, however, congruences to those primes $p$ are used for which $Q$ mod $p$ splits completely into a product of linear factors. To get the measure the fact is used that these primes have a Dirichlet-density.

In six applications results, proven in some particular cases by different techniques by Carlson [2], Inkeri [15], Ivankov [17, 18], Popken [26] and Bundschuh-Wallisser [1] are derived.

Keywords: irrationality, $Q$-linear independence.

\section{Introduction}

In [31] we used a "divisibility" method to prove a result on $\mathbb{Q}$-linear independence of values for a hypergeometric function of the form

$$
G_{Q}(x):=\sum_{n=0}^{\infty} \frac{x^{n}}{Q(1) Q(2) \cdots Q(n)},
$$

$Q \in \mathbb{Z}[x]$ a nonconstant polynomial of degree $q$ with integer coefficients and without zeros at the positive integers.

In $[32]$ we added a measure for the $\mathbb{Q}$-linear independence.

2010 Mathematics Subject Classification: primary: 11J72, 11J82; secondary: 11K60, $11 \mathrm{~J} 25,11 \mathrm{~A} 07$ 
In the present paper the general hypergeometric series

$$
{ }_{R} G_{Q}(x):=\sum_{n=0}^{\infty} \frac{R(1) R(2) \cdots R(n)}{Q(1) Q(2) \cdots Q(n)} x^{n}
$$

is considered; $R$ and $Q$ are polynomials in $\mathbb{Z}[x]$ of degree $r$ and $q$ respectively, with $r<q$ and the property $R(n) \cdot Q(n) \neq 0$ for $n=1,2,3, \ldots$ It is proved:

Theorem 1. Let $\kappa_{R}$ and $\kappa_{Q}$ be the numbers of irreducible components in the factorization over $\mathbb{Q}$ of $R$ and $Q$ respectively. Assume $\kappa_{Q}>\kappa_{R}$. Let $\alpha_{1}, \ldots, \alpha_{h}$ be any distinct non-zero rational numbers. Then the following measure of $\mathbb{Q}$-linear independence holds: For any $\varepsilon>0$ there exists an integer $H_{0}(\varepsilon)$ such that for any non-zero vector

$$
\left(s_{0}, s_{1}^{(0)}, \ldots, s_{h}^{(0)}, \ldots, s_{1}^{(q-1)}, \ldots, s_{h}^{(q-1)}\right) \in \mathbb{Z}^{h q+1} \backslash \overrightarrow{0}
$$

of height $H:=\max \left\{s_{0}, \max _{\substack{1 \leqslant j \leqslant h \\ 0 \leqslant \nu \leqslant q-1}}\left|s_{j}^{(\nu)}\right|\right\}>H_{0}(\varepsilon)$, we have

$$
\left|s_{0}+\sum_{j=1}^{h} \sum_{\nu=0}^{q-1} s_{j}^{(\nu)}{ }_{R} G_{Q}^{(\nu)}\left(\alpha_{j}\right)\right|>H^{-\left((h q+1) \frac{q-r}{\kappa_{Q}-\kappa_{R}}-1\right)-\varepsilon} .
$$

For an illustration of this theorem we give some applications.

Theorem 2. For a constant polynomial $R(x)$ different from zero, Theorem 1 contains all results which are mentioned in ([31], [32]).

Theorem 3 (compare Inkeri [15] and Li-Markov [16]). Let $w^{2}$ be a rational number different from zero. Then $\cos w$ is irrational. In addition holds: For all pairs $\left(s_{0}, s_{1}^{0}\right) \in \mathbb{Z}^{2} \backslash \overrightarrow{0}$ with height $H=\max \left\{\left|s_{0}\right|,\left|s_{1}^{0}\right|\right\}$ and for all real numbers $\varepsilon>0$ there is a $H_{0}(\varepsilon)$ such that for $H>H_{0}(\varepsilon)$ we have $\left|s_{0}+s_{1}^{(0)} \cos w\right| \geqslant H^{-2-\varepsilon}$.

Hint for the proof: Use the hypergeometric series

$$
\cos \sqrt{z}=\sum_{n=0}^{\infty} \frac{(-1)^{n} z^{n}}{(2 n) !}=\sum_{n=0}^{\infty} \frac{1}{n !}\left(\frac{-z}{2}\right)^{n}={ }_{x} G_{1}\left(\frac{-z}{2}\right) .
$$

Theorem 4 (compare Ivankov [17]). Let $R(x)$ and $Q(x)$ be polynomials of the form

$$
\begin{aligned}
& R(x)=\left(x+a_{1}\right)\left(x+a_{2}\right) \ldots\left(x+a_{r}\right) \\
& Q(x)=\left(x+b_{1}\right)\left(x+b_{2}\right) \ldots\left(x+b_{q}\right), \quad q>r .
\end{aligned}
$$

Let $R(n) \cdot Q(n) \neq 0$ for all $n \in \mathbb{N}$ and let $a_{1}, \ldots, a_{r}, b_{1}, \ldots, b_{q}$ be rational numbers with the property $a_{i}-b_{j} \notin\{0, \pm 1, \pm 2, \ldots\}$ for all pairs $(i, j), i \in\{1, \ldots, r\}, j \in$ $\{1, \ldots, q\}$. Then we have, with the notations in Theorem 1,

$$
\left|s_{0}+\sum_{j=1}^{h} \sum_{\nu=0}^{q-1} s_{j}^{(\nu)}{ }_{R} G_{Q}^{(\nu)}\left(\alpha_{j}\right)\right|>H^{-q h-\gamma(\ln \ln H)^{-1}},
$$

$\gamma$ is a real number dependent only on $R, Q$ and $\alpha_{1}, \ldots, \alpha_{h}$. 
Theorem 5 (see Theorem 1 in Ivankov [18]). For $R(x)=x^{2}, Q(x)=$ $\left(x^{2}+1\right)\left(x^{2}-2\right)\left(x^{2}-3\right)\left(x^{2}-6\right)$ we have: For any $\varepsilon>0$ there exists $H_{0}(\varepsilon)$ such that for $H>H_{0}(\varepsilon)$ with $H:=\max \left\{\left|s_{0}\right|,\left|s_{1}\right|, \ldots,\left|s_{8}\right|\right\} \in \mathbb{Z}^{9} \backslash \overrightarrow{0}$ the following estimation holds:

$$
\left|s_{0}+\sum_{j=1}^{8} s_{j R} G_{Q}^{(j-1)}(1)\right|>H^{-26-\varepsilon} .
$$

Remark. Without any change in the proof of Theorem 1 in [32] we can get, using a result on polynomial congruences which I owe to Schinzel (see Section 4), the following

Theorem 6. Let $R$ and $Q$ be polynomials with integer coefficients. $R$ should not be of the first degree and $R$ should be irreducible over the splitting field of $Q$. As in Theorem 1 let $\kappa_{Q}>\kappa_{R}$ and let $\alpha_{1}, \ldots, \alpha_{h}$ be distinct nonzero rational numbers. Then the same estimation as in Theorem 1 is valid for the hypergeometric series ${ }_{R} G_{Q}(x)$.

An explicit application of this Theorem is for example: Let $R(x)=x^{2}+x+1$, $Q(x)=(x+1)\left(x^{2}+1\right)$. Here we have $\kappa_{Q}=2, \kappa_{R}=1$. With $\alpha \in \mathbb{Q} \backslash\{0\}$ we get from Theorem 6

$$
\left|s_{0}+\sum_{\nu=0}^{2} s_{j}^{(\nu)}{ }_{R} G_{Q}^{(\nu)}(\alpha)\right| \geqslant H^{-3-\varepsilon}, \quad H>H_{0}(\varepsilon) .
$$

Remark. From the approximation-theorem of Dirichlet follows that the exponent $3+\varepsilon$ is best possible.

Proof of Theorem 1. To prove Theorem 1, we follow the lines of [31] or [32]. Some changes are necessary because of the application of Hadamard's multiplication of power series. But the essential idea is again to use "divisibility properties" of the coefficients of the approximation polynomial. This method was applied at several places in Niven's [24] monograph on irrational numbers. To avoid repetitions we give, aside Section 5 on divisibility propeties, only a sketch how the nine Sections of [32] have to be renewed.

Acknowledgments. I thank Peter Bundschuh for reading a former manuscript of the paper and for helpful suggestions.

\section{New or renewed sections compared to [31] or [32].}

\subsection{On Hadamard's multiplication $(*)$}

We use the definitions

$$
\begin{aligned}
{ }_{i} P_{m}^{N}(z) & :=a_{m, i} z^{m}+a_{m+1, i}, z^{m+1}+\ldots+a_{N, i} z^{N} \in \mathbb{C}_{m}^{N}[z], \quad i=1,2 \\
{ }_{1} P_{m}^{N}(z) *{ }_{2} P_{m}^{N}(z) & :=a_{m, 1} \cdot a_{m, 2} z^{m}+\cdots+a_{N, 1} \cdot a_{N, 2} z^{N}
\end{aligned}
$$


For polynomials $f, P \in \mathbb{C}_{m}^{N}[z]$ and a general polynomial $Q \in \mathbb{C}[z]$ we can show with $\delta:=z \frac{d}{d z}$ the relations

$$
\begin{aligned}
f(z) *(Q(\delta) \circ P(z)) & =Q(\delta) \circ(f(z) * P(z)) \\
\frac{1}{z}(f(z) *(Q(\delta) \circ P(z))) & =\frac{1}{z} f(z) * \frac{1}{z}(Q(\delta) \circ P(z)),
\end{aligned}
$$

and for the $\nu$ th-derivative we have

$$
\begin{gathered}
(f(z) * P(z))^{(\nu)}=f(z)^{(\nu)} * P(z)^{[\nu]}=f(z)^{[\nu]} * P(z)^{(\nu)} \\
P(z)^{[\nu]}:=p_{\nu}+p_{\nu+1} \cdot z+\ldots+p_{N} \cdot z^{N-\nu} .
\end{gathered}
$$

\subsection{The approximation polynomials $P_{\mu}(z)$}

They are defined by

$$
P_{\mu}(z):=\sum_{n=\mu}^{m} \gamma_{n} k_{n-\mu} z^{n-\mu}, \quad 0 \leqslant \mu \leqslant m ;
$$

$\gamma_{n}$ are special complex numbers which are defined later, $R, Q \in \mathbb{Z}[z]$, $\operatorname{deg} R=r<$ $q=\operatorname{deg} Q$.

$$
\begin{gathered}
k_{n}:=\frac{R(1) \cdots R(n)}{Q(1) \cdots Q(n)}, \quad n \in \mathbb{N}, \quad k_{0}:=1, \\
R(n) Q(n) \neq 0, \quad n \in \mathbb{N} .
\end{gathered}
$$

Essential is the relation

$$
Q(\delta) P_{\mu}(z)=\gamma_{\mu} Q(0)+R(\delta) z P_{\mu+1}(z), \quad 0 \leqslant \mu \leqslant m-1 .
$$

\subsection{Connection of $P_{\mu}(z)$ with the "starting polynomial" $P_{0}(z)$}

$$
\hat{R}_{\mu}(z):=\sum_{n=\mu+1}^{m} R(n-\mu) z^{n-\mu}
$$

If $\gamma_{n}=0$ for $n=0, \ldots, p-2(p \leqslant m, p$ a special prime number, see Sections 4 and 5), then we get from (2.1)

$$
Q(\delta) P_{\mu}(z)=R(\delta) z P_{\mu+1}(z), \quad 0 \leqslant \mu \leqslant p-2 .
$$

Define

$$
\hat{R}_{\mu}^{-1}(z):=\sum_{n=\mu+1}^{m} \frac{1}{R(n-\mu)} z^{n-\mu}
$$


By iteration follows from $(2.2)$

$$
\begin{aligned}
\Psi_{\nu-1}(z) *\left(\frac{1}{z} Q(\delta)\right)^{\nu} P_{0}(z) & =P_{\nu}(z), \quad 0 \leqslant \nu \leqslant p-1 \\
\Psi_{\nu-1}(z) & :=\frac{1}{z^{\nu}} \hat{R}_{0}^{-1}(z) * \cdots * \frac{1}{z} \hat{R}_{\nu-1}^{-1}(z) .
\end{aligned}
$$

With Lemma 2 in [31] or [32, (22)] we conclude: For $1 \leqslant \mu \leqslant p-1$ holds

$$
\begin{aligned}
P_{\mu}(z) & =P_{\mu-1}(z) * \hat{P}_{\mu}(z), \\
\hat{P}_{\mu}(z) & :=\sum_{\nu=0}^{\mu \cdot q} \alpha_{\nu, \mu} z^{\nu-\mu} P_{0}^{(\nu)}(z), \quad \alpha_{\nu, \mu} \in \mathbb{C} .
\end{aligned}
$$

\subsection{Two theorems on polynomial congruences}

Lemma 4.1. Let $Q$ and $R$ be two non constant polynomials with integer coefficients. Then there exist infinitely many primes $p$ of positive Dirichlet-density which split $Q$ and $R$ completely.

Remark. For the notations in Lemma 4.1 see Section 4 in [32] or see for the definitions and proofs the paper of Gerst and Brillhart [8] (especially Section 5). A good introduction to this section is Hasse [11]. Similar results are in Nagell $[21],[22]$ or in Fjellstedt [6].

Lemma 4.2. Let the polynomial $R$ of Lemma 4.1 not be of first degree and let $R$ be irreducibel over the splitting field of $Q$. Then there exist infinitely many primes of positive Dirichlet-density such that $Q$ factors modulo $p$ into a product of linear factors and the congruence $R(x) \equiv 0(\bmod p)$ has no solution.

Proof. Schinzel [27].

Remark. For the algebraic theory of Dirichlet-densitiy compare [23], chap. VII, $\S 13$.

\subsection{Some divisibility properties of the values $P_{\mu}\left(\alpha_{j}\right)$ (see 2. in [32]) in case of special starting polynomials $P_{0}(z)$}

Let $p$ be a prime statisfying Lemma 4.1. With such primes $p$ we consider two kinds of approximation polynomials $P_{0}(z)$ :

$$
\begin{aligned}
& \text { a) } P_{0}(z):=z^{p-1} \sum_{j=1}^{h}\left(z-\alpha_{j}\right)^{p \cdot q} \\
& \text { в) } P_{0}(z):=z^{p}\left(z-\alpha_{j_{0}}\right)^{(p-1) q+\nu_{0}} \prod_{\substack{j=1 \\
j \neq j_{0}}}^{h}\left(z-\alpha_{j}\right)^{p \cdot q}, \\
& \quad 0 \leqslant \nu_{0}<q, 1 \leqslant j_{0} \leqslant h .
\end{aligned}
$$


Let $R^{*}$ be defined by

$$
R^{*}:=R(1) R(2) \cdots R(m),
$$

where $m$ is the degree of $P_{0}(z)$, namely

$$
\begin{array}{ll}
\text { a) } & m=h p \cdot q+p-1=p(h q+1)-1 \\
\text { в) } & m=h p \cdot q+p-q+\nu_{0}
\end{array}
$$

For the divisibility properties we investigate the polynomials

$$
R^{*} P_{\mu}(z)=\sum_{n=\mu}^{m} R^{*} \gamma_{n} k_{n-\mu} z^{n-\mu}, \quad 0 \leqslant \mu \leqslant m .
$$

As in Remark iii) of [31], we can show that the rational numbers $\alpha_{j}(1 \leqslant j \leqslant h)$ can be chosen to be integers. From (2.4) and the Taylor-development at zero of $P(z)$ (see the beginning of Section 2) we can conclude that the coefficients $\gamma_{n} k_{n}$ are rational integers.

We have to look for the exponent $\gamma$ of the prime number $p$ with the divisibility property $p^{\gamma} \mid R^{*} P_{\mu}\left(\alpha_{j}\right)$. From the Taylor development at zero of $P_{0}(z)=$ $\sum_{n=0}^{m} \gamma_{n} k_{n} z^{n}$ follows in case

$$
\begin{array}{lll}
\text { a) } & P_{\mu}(0)=P_{0}^{(\mu)}(0)=\gamma_{\mu}=0 & \text { for } \mu<p-1 \\
\text { в) } & P_{\mu}(0)=P_{0}^{(\mu)}(0)=\gamma_{\mu}=0 & \text { for } \mu<p .
\end{array}
$$

Using (2.3) and the definition of the starting polynomials in (2.4) we get

$$
\begin{aligned}
& P_{\mu}^{(\rho)}\left(\alpha_{j}\right)=0 \quad \text { for } \mu \leqslant p-1, \quad\left\{\begin{array}{l}
1 \leqslant j \leqslant h \\
0 \leqslant \rho<q
\end{array} \quad j \neq j_{0}\right\} \\
& P_{\mu}^{(\rho)}\left(\alpha _ { j _ { 0 } ) } = 0 \quad \text { for } \quad \left\{\begin{array}{ll}
0 \leqslant \mu<p-1, & 0<\rho<q \\
\mu=p-1, & \rho<\nu_{0}
\end{array}\right.\right. \\
& P_{p-1}^{\left(\nu_{0}\right)}\left(\alpha_{j_{0}}\right)=\frac{1}{R\left(\nu_{0}+1\right) \cdots R\left(\nu_{0}+p-1\right)} \hat{P}_{p-1}^{\left(\nu_{0}\right)}\left(\alpha_{j_{0}}\right) \neq 0,
\end{aligned}
$$

$(\operatorname{see}(2.3))$.

The prime number $p$ is chosen in such a way that the polynomials $Q$ and $R$ split completely modulo $p$. Then we have

$$
Q(x) \equiv \bar{Q}(x)=\left(x-\bar{\alpha}_{1}\right)^{r_{1}}\left(x-\bar{\alpha}_{2}\right)^{r_{2}} \cdots\left(x-\bar{\alpha}_{t}\right)^{r_{t}}(\bmod p)
$$

with integers $\bar{\alpha}_{i}, 0 \leqslant \bar{\alpha}_{i}<p$, and natural numbers

$$
r_{i}, \quad 1 \leqslant i \leqslant t, \quad r_{1}+r_{2}+\cdots+r_{t}=q=\operatorname{deg} Q .
$$


For a product of the form

$$
\bar{Q}(n-\mu+1) \cdots \bar{Q}(n)=\prod_{h=0}^{\mu-1}\left(n-h-\bar{\alpha}_{1}\right)^{r_{1}} \cdots\left(n-h-\bar{\alpha}_{t}\right)^{r_{t}}
$$

with $n \geqslant \mu \geqslant p$ the set $\{n-(\mu-1), \ldots, n\}$ contains at least one complete system of residues modulo $p$. Therefore, in such a set, there is at least one integer $\ell_{i}$ with $(n-\mu-1) \leqslant \ell_{i} \leqslant n$ and the property $\ell_{i}-\alpha_{i} \equiv 0(\bmod p)$. That's why we have $p^{r_{i}} \mid \prod_{h=0}^{\mu-1}\left(n-h-\bar{\alpha}_{i}\right)^{r_{i}}$ and it follows

$$
p^{q} \mid Q(n-\mu+1) \cdots Q(n), \quad \mu \geqslant p .
$$

For the polynomial $R$ we get in the same way with $r=\operatorname{deg} R$ :

$$
p^{r} \mid R(n-\mu+1) \cdots R(n) .
$$

We can derive for such a prime $p$ from (2.5)

$$
p^{\left[\frac{m}{p}\right] r} \mid R^{*}
$$

and from (2.6) in case $\alpha$ ) for $\delta_{1} \in \mathbb{N}$ with $p^{\delta_{1}} \mid R^{*}$

$$
\delta_{1}=\left[h q r+r-\frac{r}{p}\right] .
$$

For $p \gg r$ follows $\delta_{1}=h q r+r-1$.

To find the exponent $\delta_{2}$ with

$$
p^{\delta_{2}} \mid R^{*} P_{\mu}\left(\alpha_{j}\right), \quad 0 \leqslant \mu \leqslant m, 1 \leqslant j \leqslant h,
$$

we have to estimate $\delta_{3} \in \mathbb{N}$ with

$$
p^{\delta_{3}} \mid R^{*} \gamma_{n} k_{n-\mu} \quad \text { for } m \geqslant n \geqslant \mu \geqslant p .
$$

It is

$$
\begin{aligned}
R^{*} \gamma_{n} k_{n-\mu} & =R^{*} \gamma_{n} k_{n} \frac{k_{n-\mu}}{k_{n}} \\
& =\gamma_{n} k_{n} R(1) \cdots R(n-\mu) Q(n-\mu+1) \cdots Q(n) R(n+1) \cdots R(m) .
\end{aligned}
$$

From $(2.6) \alpha),(2.14)$ and $(2.15)$ we get for $\delta_{3}$ in (2.19), as $\mu \geqslant p$,

$$
\left[h q r-\frac{r}{p}+q\right] \leqslant \delta_{3}
$$

and from this we conclude with (2.7) for $p \gg r$

$$
p^{h q r+q-1} \| R^{*} P_{\mu}\left(\alpha_{j}\right), \quad 1 \leqslant j \leqslant h, \mu \geqslant p,\left(a^{\kappa} \| b:=a^{\kappa} \mid b \text { but } a^{\kappa+1} \nmid b\right) .
$$


In the same way we can show with $(2.20)$

$$
p^{h q r+q-1} \| R^{*} P_{\mu}(0), \quad \mu \geqslant p-1 .
$$

In case $\beta$ we have to use the relations $(2.4) \beta),(2.6) \beta),(2.20),([32],(32))$ and $([32]$, (49)). We get

$$
\begin{aligned}
& p^{\delta_{4}} \mid R^{*} P_{\mu}(0) \quad \text { with } \delta_{4}=[h q r+q], \text { or }(\text { for } p \gg) \\
& p^{h q r+q} \| P^{*}(0) .
\end{aligned}
$$

Further

$$
\begin{aligned}
& p^{h q r+q-1} \| R^{*} P_{p-1}^{\left(\nu_{0}\right)}\left(\alpha_{\left.j_{0}\right)}\right. \\
& p^{h q r+q} \mid R^{*} P_{\mu}^{(\rho)}\left(\alpha_{j}\right), \quad j \neq j_{0}, \quad 1 \leqslant j \leqslant h, \mu \geqslant p, \rho<q . \\
& p^{h q r+q} \mid R^{*} P_{p-1}^{(\rho)}\left(\alpha_{j_{0}}\right), \quad \nu_{0}<\rho<q .
\end{aligned}
$$

\subsection{A further application of the method of Hilbert-Perron-Skolem} (vgl. ([29], page 93))

In [31] or $\left([32], 2\right.$.) we use the polynomial $P^{*}$,

$$
P^{*}(x):=\sum_{\mu=0}^{m} P_{\mu}(x),
$$

to show that the linear form

$$
\Lambda:=s_{0}+\sum_{j=1}^{h} \sum_{\nu=0}^{q-1} s_{j}^{(\nu)}{ }_{R} G_{Q}^{(\nu)}\left(\alpha_{j}\right)
$$

can be approximated by

$$
\Lambda^{*}:=s_{0} P^{*}(0)+\sum_{j=1}^{h} \sum_{\nu=0}^{q-1} s_{j}^{(\nu)} P^{*(\nu)}\left(\alpha_{j}\right) .
$$

It is essential to show that $\Lambda^{*}$ is not zero. The proof follows in Section 7 . Then we give a lower estimation of $\Lambda^{*}$ and use

$$
\left|P^{*}(0) \cdot \Lambda\right| \geqslant \frac{1}{2}\left|\Lambda^{*}\right|>0
$$

to estimate the form in question $\Lambda$. To get a measure for $Q$-linear independence of the number 1 and the values ${ }_{R} G_{Q}^{(\nu)}\left(\alpha_{j}\right), j=1, \ldots, h$, we have to estimate the absolute values of $P^{*}(0)$ and $\Lambda^{*}$. 


\subsection{The approximation-form $\Lambda^{*}$ is different from zero}

We have by definition

$$
\Lambda^{*}:=s_{0} P^{*}(0)+\sum_{j=1}^{h} \sum_{\nu=0}^{q-1} s_{j}^{(\nu)} P^{*(\nu)}\left(\alpha_{j}\right) .
$$

$\alpha)$ This case is defined by

$$
p \nmid s_{0}, \quad p \mid s_{j}^{(\nu)}, \quad 1 \leqslant j \leqslant h, 0 \leqslant \nu<q .
$$

From (2.33) and the divisibility properties in Section 5 we get for $\kappa=h q r+q$

$$
p^{\kappa} \mid\left(R^{*} \Lambda^{*}-s_{0} R^{*} P^{*}(0)\right) \quad \text { and } \quad p^{\kappa} \nmid s_{0} R^{*} P^{*}(0),
$$

therefore $\Lambda^{*} \neq 0$.

$\beta)$ In this case we have (see the choice of $p \in \mathbb{P}$ in $([32],(26) \beta)$ )

$$
\begin{aligned}
& p \nmid s_{j_{0}}^{\left(\nu_{0}\right)}, \quad p \mid s_{j}^{(\nu)}, \quad\left(1 \leqslant j \leqslant h, \nu_{0}<\nu<q\right), \\
& p \mid s_{j}^{\left(\nu_{0}\right)}, \quad j_{0}<j<h .
\end{aligned}
$$

For all other coefficients $s_{j}^{(\nu)}$ no assumption concerning the " $p$-divisibility" is made. With (2.34), (2.8)-(2.10) and (2.24)-(2.28) we get

$$
\begin{aligned}
& p^{h q r+q} \mid\left(R^{*} \Lambda^{*}-s_{j_{0}}^{\left(\nu_{0}\right)} R^{*} P^{*\left(\nu_{0}\right)}\left(\alpha_{j_{0}}\right)\right) \\
& p^{h q r+q} \nmid s_{j_{0}}^{\left(\nu_{0}\right)} R^{*} P^{*\left(\nu_{0}\right)}\left(\alpha_{j_{0}}\right) .
\end{aligned}
$$

These two relations lead again to $\Lambda^{*} \neq 0$.

\subsection{A lower estimate for $\Lambda^{*}$}

Let $t$ be a prime number and let

$$
\mu_{R, t}:=|\{x \in \mathbb{Z}, 0 \leqslant x<t, R(x) \equiv 0 \bmod t\}| \quad \text { and similarly } \mu_{Q, t} .
$$

According to Nagell [21] we have the estimates:

$$
\sum_{t \leqslant x} \mu_{R, t} \frac{\log t}{t}=\kappa_{R} \log x+\mathrm{O}(1)
$$

and analogus for $R$ replaced by $Q$.

With

$$
\tilde{B}_{p}:=\prod_{t \in \mathbb{P}} t^{\left(\mu_{Q, t}-\mu_{R, t}\right)\left[\frac{p}{t}\right]}
$$

we can prove as in $[32,7$.]

$$
\tilde{B}_{p} \mid P(0) \quad \text { and } \quad \tilde{B}_{p} \mid P_{\mu}\left(\alpha_{j}\right), \quad(0 \leqslant \mu \leqslant m, 1 \leqslant j \leqslant h),
$$

and we get,

$$
\log \left|\Lambda^{*}\right| \geqslant\left(\kappa_{Q}-\kappa_{R}\right) p \log p+\mathrm{O}(\mathrm{p}) .
$$




\subsection{Estimates of the remainder terms $\Delta^{(\nu)}\left(\alpha_{j}\right)$}

$$
\Delta(x):=P^{*}(x)-P^{*}(0)_{R} G_{Q}(x) .
$$

As in $[32,8$.$] we can get the relations$

$$
\left|\Delta^{(\nu)}\left(\alpha_{j}\right)\right| \leqslant c_{3}^{p}, \quad 0 \leqslant \nu \leqslant q-1,1 \leqslant j \leqslant h,
$$

where $c_{3}$ is a constant independent of $p$.

\subsection{Proof of Theorem 1}

The key is the relation between the original form

$$
\Lambda:=s_{0}+\sum_{j-1}^{h} \sum_{\nu=0}^{q-1} s_{j}^{(\nu)}{ }_{R} G_{Q}^{(\nu)}\left(\alpha_{j}\right)
$$

and the approximation-form $\Lambda^{*}$ :

$$
\Lambda^{*}:=s_{0} P^{*}(0)+\sum_{j-1}^{h} \sum_{\nu=0}^{q-1} s_{j}^{(\nu)} P^{*(\nu)}\left(\alpha_{j}\right)
$$

$\Lambda$ and $\Lambda^{*}$ are connected by

$$
\Lambda^{*}=P^{*}(0) \Lambda+\sum_{\nu=0}^{p-1} \sum_{j=1}^{h} s_{j}^{(\nu)} \Delta^{(\nu)}\left(\alpha_{j}\right) .
$$

Using the estimates in Sections 8 and 9 we have, with $H:=\max _{\nu, j}\left(\left|s_{j}^{(\nu)}\right|\right)$,

$$
\begin{aligned}
\left|P^{*}(0) \Lambda\right| & \geqslant\left|\Lambda^{*}\right|-\sum_{\nu=0}^{q-1} \sum_{j-1}^{h}\left|s_{j}^{(\nu)} \Delta^{(\nu)}\left(\alpha_{j}\right)\right| \\
& \geqslant \exp \left(\left(\kappa_{Q}-\kappa_{R}\right) p \log p+O(p)\right)-H \exp \left(p c_{4}\right) .
\end{aligned}
$$

This leads to the estimate in Theorem 1 in the same way as in $([32], 9$.$) .$

\section{References}

[1] P. Bundschuh and R. Wallisser, Some arithmetical applications of Newton's interpolation series, Analysis (Munich) 32 (2012), 1001-1018.

[2] F. Carlson, Sur une propriété arithmétique de quelques functions entières, Ark. Mat. Astr. Fys. 25A (1935), 1-13.

[3] R. Dedekind, Über den Zusammenhang zwischen der Theorie der Ideale und der höheren Kongruenzen, Abh. Akad. Wiss. Göttingen, (1878). 
[4] N.I. Fel'dman and Yu.V. Nesterenko, Transcendental Numbers, Number Theory IV, Encycl. of Math. Sc. 44, Springer 1998.

[5] G. Frobenius, Über Beziehungen zwischen den Primidealen eines algebraischen Körpers und den Substitutionen einer Gruppe, Berl. Ber., 1886, I.S. 683-703.

[6] L. Fjellstedt, Bemerkungen über gleichzeitige Lösbarkeit von Kongruenzen, Ark. Mat. 3 (1955), 193-198.

[7] A.I. Galochkin, On effective bounds for certain linear forms, New Advances in Transcendence Theory, Cambridge Univ. Press, Cambridge (1988), 207-214.

[8] I. Gerst and J. Brillhart, On the prime divisors of polynomials, Amer. Math. Monthly 78 (1971), 250-266.

[9] J. Hadamard, Essai sur l'étude des fonctions donneés par leur dévelopement de Taylor, Math. Pures Appl. Sér. 48 (1892).

[10] H. Hasse, Bericht über neuere Untersuchungen und Probleme aus der Theorie der algebraischen Zahlkörper, Teil II: Reziprozitätsgesetze. Jber. Deutsch. Math. Verein 36 (1930).

[11] H. Hasse, Über das Problem der Primzerlegung in galoischen Zahlkörpern, Vortrag in der Berliner Math. Gesellschaft (1952), Preprint etenda.

[12] Ch. Hermite, Sur la fonction exponentielle, C.R. Acad. Sci, Paris, Sér. A. (1873).

[13] D. Hilbert, Über die Transzendenz der Zahlen e und $\pi$, Math. Ann. 43 (1893), 216-219.

[14] A. Hurwitz, Beweis der Transzendenz der Zahl e, Göttinger Nachrichten Nr. 4 (1893).

[15] K. Inkeri, On the irrationality of certain values of elementary transcendental functions, Nieuw. Arch. Wisk. (3) 24 (1976), 226-230.

[16] Zhou Li and L. Markov, Recurrent proofs of the irrationality of certain trigomometric values Amer. Math. Monthly 117 (2010), 360-362.

[17] P.L. Ivankov, On arithmetic properties of the values of hypergeometric functions, Math. USSR Sb. 72 (1992), no.1, 267-286.

[18] P.L. Ivankov, The values of certain hypergeometric functions with irrational parameters, Russian Mathematics (Izv. Vuz) 51 (2007), no. 7, 45-49.

[19] L. Kronecker, Vorlesungen über Zahlentheorie, Bd. 1, Teubner 1901, Reprint Springer 1978.

[20] F. Lindemann, Über die Zahl $\pi$, Math. Ann. 20 (1882), 213-225.

[21] T. Nagell, Généralisation d'un théorème de Tchebycheff, Math. Pures Appl. ( $8^{e}$ série) 4 (1921), 343-356.

[22] T. Nagell, Sur les diviseurs premiers de polynomes, Acta Arithmetica XV (1969), 235-244.

[23] J. Neukirch, Algebraische Zahlentheorie, Springer-Verlag 1992, Nachdruck 2007.

[24] I. Niven, Irrational numbers, The Carus Mathematical Monographs no. 11 (1956).

[25] O. Perron, Irrationalzahlen, Chelsea, New York, 1951.

[26] J. Popken, Über arithmetische Eigenschaften analytischer Funktionen, Dissertation Amsterdam 1935. 
[27] A. Schinzel, Private communication (2006).

[28] A.B. Shidlowskii, Transcendental Numbers, De Gruyter Studies in Mathematics 12, Berlin-New York 1989.

[29] Th. Skolem, Some theorems on irrationality and linear independence, Skand. Kongr. 11, Trondheim 1949, 77-98.

[30] V.G. Sprindzuk, The irrationality of the values of certain transcendental functions, Akad. Nauk SSSR Ser. Mat. 32 (1968).

[31] R. Wallisser, Linear independence of values of a certain generalisation of the exponential function - a new proof of a theorem of Carlson, Journal de Theórie des Nombres de Bordeaux 17 (2005), 381-396.

[32] R. Wallisser, A measure for the linear independence of values of a certain generalization of the exponential function, Functiones et Approximatio XXXV (2006), 339-357.

[33] K. Weierstrass, Über die Ludolphsche Zahl, Sitzungsber. Preuss. Akad. Wiss (1885), 1067-1085.

Address: Rolf Wallisser: Fakultät für Mathematik und Physik, der Albert-Ludwigs-Universität Freiburg, Abteilung für Reine Mathematik, Eckerstr. 1, D-79104 Freiburg, Germany.

E-mail: rolf.wallisser@mesonet.de

Received: 18 April 2012; revised: 14 June 2013 